\title{
Variants of neurogenin 3 gene are not associated with Type II diabetes in Japanese subjects
}

 \\ ${ }^{1}$ Department of Metabolic Diseases, Graduate School of Medicine, University of Tokyo, Tokyo, Japan \\ ${ }^{2}$ Department of Medical Genetics, School of Medicine, Chiba University, Chiba, Japan \\ ${ }^{3}$ Department of Geriatric Medicine, Osaka University Medical School, Osaka, Japan \\ ${ }^{4}$ Hiroshima Atomic Bomb Casualty Council Health Management Center, Hiroshima, Japan
}

\section{Abstract}

Aims/hypothesis. Neurogenin 3 (ngn3) is a transcription factor expressed in the endocrine precursor cells of the pancreas. It has recently been reported that ngn3-deficient mice show absence of pancreatic endocrine cells and die of postnatal diabetes. The purpose of this investigation was to screen for polymorphisms of the ngn3 gene and to test whether these polymorphisms are associated with Type II (non-insulin-dependent) diabetes mellitus in the Japanese subjects.

Methods. We screened ngn3 gene and upstream region by direct sequencing and estimated the prevalence of polymorphisms in 197 patients with Type II (non-insulin-dependent) diabetes mellitus and 216 control subjects.
Results. We identified four novel polymorphisms, Ser199Phe $(596 C / T),-43 i n s C A,-983 C / T$ and $-1822 G / A$. In an association study the allelic frequencies of the major allele of these four polymorphisms were $0.721,0.914,0.912$ and 0.530 in diabetic patients, respectively, and $0.694,0.905,0.917$ and 0.537 in control subjects, respectively.

Conclusion/interpretation. Mutations and polymorphisms of $n g n 3$ gene are not significantly associated with Type II (non-insulin-dependent) diabetes mellitus in the Japanese subjects. [Diabetologia (2001) 44: 241-244]

Keywords Neurogenin 3, transcription factor, Type II diabetes mellitus, polymorphisms, basic helix-loophelix.
Type II (non-insulin-dependent) diabetes mellitus is a polygenic disorder characterized by insulin secretory defects or insulin resistance or both, but the precise underlying genetic factors are largely not known. Recently, mutations and polymorphisms of transcription factors expressed in pancreatic endocrine cells have been reported to be related to the development of diabetes mellitus. Hepatocyte nuclear factor- $1 \alpha(H N F-$ $1 \alpha$ ), HNF-4 $\alpha$ and insulin promoter factor-1 (IPF-1) genes have been reported to be associated with MODY (maturity-onset diabetes of the young) [1].

Received: 23 June 2000 and in revised form: 11 October 2000

Corresponding author: T. Kadowaki, MD, PhD, 7-3-1, Hongo, Bunkyo-ku, Tokyo 113-8655, Japan

Abbreviations: HNF, hepatocyte nuclear factor; MODY, maturity-onset diabetes of the young; bHLH, basic helix-loop-helix.
Variants of IPF-1 [2, 3] and NeuroD [4] have also been reported to be related to the development of Type II diabetes.

Neurogenins are a set of basic helix-loop-helix (bHLH) transcription factors which are essential for the determination of neuronal tissues during embryonic development. Neurogenin 1 and neurogenin 2 are essential for the determination of sensory lineages in the peripheral nervous system. Neurogenin 3 (ngn3) is expressed not only in restricted domains of the developing central nervous system but also in the endocrine precursor cells of the pancreas [5]. It has been shown that $n g n 3$-deficient mice lack pancreatic endocrine cells and die of postnatal diabetes [6]. It has therefore been suggested that ngn3 is essential for the development of pancreatic endocrine cells. We thus considered the possibility that ngn 3 could be a candidate gene responsible for Type II diabetes. 
Table 1. Clinical characteristics of subjects

\begin{tabular}{lll}
\hline & $\begin{array}{l}\text { Type II diabetic } \\
\text { patients }\end{array}$ & $\begin{array}{l}\text { Control } \\
\text { subjects }\end{array}$ \\
\hline$n$ & 197 & 216 \\
Men/Women & $130 / 67$ & $105 / 111$ \\
Age (years) & $57.5 \pm 12.6$ & $67.2 \pm 7.8$ \\
$\begin{array}{l}\text { Duration of hyperglycaemia } \\
\text { (years) }\end{array}$ & $11.3 \pm 8.4$ & - \\
BMI $\left(\mathrm{kg} / \mathrm{m}^{2}\right)$ & $24.5 \pm 4.8$ & $23.0 \pm 2.8$ \\
HbA & $(\%)$ & $5.2 \pm 0.2$ \\
Treatment & $8.0 \pm 2.0$ & \\
Insulin/OHA/diet $[n(\%)]$ & $53(26.9) / 97(49.2) /$ & - \\
& $47(23.9)$ & \\
\hline
\end{tabular}

OHA: Oral hypoglycaemic agents

\section{Subjects and methods}

Subjects. Subjects with Type II diabetes were recruited from the Department of Metabolic Diseases, University of Tokyo Hospital, and control subjects were recruited from the Hiroshima Atomic Bomb Casualty Council Health Management Center. All the subjects enrolled in this study were ethnic Japanese. Type II diabetes was diagnosed according to the criteria of the World Health Organisation [7]. Control subjects had to meet all of the following three criteria: 1) age of more than 60 years, 2) $\mathrm{HbA}_{1 \mathrm{c}}$ of less than $6.0 \%, 3$ ) no family history of Type II diabetes. The characteristics of the patients with Type II diabetes and control subjects are shown in Table 1. A total of 81 of the diabetic patients and 20 of the control subjects were screened for polymorphisms from among the 197 patients with Type II diabetes and 216 control subjects recruited for the association study. All the participants gave their written informed consent before participating in the study, and the protocol was approved by the ethics committee of the University of Tokyo Hospital.

Nucleotide numbering. The A of ATG of the initiator Met codon of the sequence is denoted as nucleotide +1 .

Screening for mutations in the ngn3 gene. We conducted direct sequencing of the entire coding region, 5'- and 3'-untranslated regions (UTR), as well as intron 1 of $n g n 3$ in 81 Japanese patients with Type II diabetes and 20 control subjects, based on the sequence reported in GenBank (accession number AJ133776). We also performed direct sequencing of $2.4 \mathrm{~kb}$ of upstream region of $n g n 3$ in 20 Japanese patients with Type II diabetes and 20 control subjects, based on the draft sequence reported in GenBank (accession number AC011010). These regions of the ngn3 gene were amplified by polymerase chain reaction (PCR) using the nine sets of primers. The segments were amplified using specific primers as follows: segment 1 (nucleotide position -318 86): forward, 5'-CGGACCCCATTCTCTCTTCT-3', reverse, 5'-ACTTCGTCTTCCGAGGCTCT-3'; segment 2 a (nucleotide position -29 272): forward, 5'-CTTACCСТTCCCTCTGACCC-3', reverse, 5'-CGGTCGTTGGCCTTCTTTCG-3'; segment 2 b (nucleotide position 220 529): forward, 5'-GAGTTGGCACTGAGCAAGCAG3', reverse, 5'-AGACTGGGGAGTAGAGGGAC-3'; segment 2c (nucleotide position $421 \sim 821$ ): forward, 5'-ATAGCGGACCACAGCTTGTA-3', reverse, 5'-GAATGAACCCAGCCTACCG-3'; segment up1 (nucleotide position $-799 \sim-171$ ): forward, 5'-CAAGCCACCTCCСТCACTAA-
3', reverse, 5'-CTACCGGCGCAAAAGAATAG-3'; segment up2 (nucleotide position -1306 -678): forward, 5'-CGACAGAAGGAGACCGTTTT-3', reverse, 5'-CGGCTTTGCTCCTAGCCTAT-3'; segment up3 (nucleotide position -1791 -1177): forward, 5'-GTTTCCAGAGAGCGCTACCA-3', reverse, 5'-AGGAACCGTGACAGGAAGAG-3'; segment up4 (nucleotide position -2284 -1640): forward, 5'GAAGGAGCCTGCTCACAGAG-3', reverse, 5'-TAGTCCTCCGGTCCAATCAC-3'; segment up5 (nucleotide position -2729 -2159): forward, 5'-AGCAGCTTGAATGGACAGTG-3', reverse, 5'-GGCAAATTTTCCTCCAAGG-3'. PCR was carried out in a $20-\mu 1$ reaction mixture, containing $20 \mathrm{ng}$ template DNA, $1.5 \mathrm{mmol} / \mathrm{l} \mathrm{MgCl}_{2}, 200 \mu \mathrm{mol} / \mathrm{l}$ of each $\mathrm{dNTP}, 500 \mathrm{nmol} / 1$ of each primer, and $0.5 \mathrm{U}$ Taq polymerase in the buffer supplied by the manufacturer. For amplification of segments 1, 2a, 2b, $2 \mathrm{c}$ and up1, $5 \%$ DMSO was added. After initial denaturation at $95^{\circ} \mathrm{C}$ for $5 \mathrm{~min}, 35$ cycles of $30 \mathrm{sec}$ at $94^{\circ} \mathrm{C}, 30 \mathrm{sec}$ at $58^{\circ} \mathrm{C}, 30 \mathrm{sec}$ at $72^{\circ} \mathrm{C}$ were run, followed by an additional $7 \mathrm{~min}$ at $72^{\circ} \mathrm{C}$ after the last cycle. The PCR products were purified and sequenced on an ABI PRISM $310 \mathrm{Ge}$ netic Analyzer using the BigDye Terminator (Perkin Elmer, Foster City, Calif., USA).

Allelic frequency study. Allelic frequency study of the Ser199Phe $(596 \mathrm{C} / \mathrm{T})$ and the $-983 \mathrm{C} / \mathrm{T}$ polymorphisms was carried out by restriction fragment length polymorphism analysis. For the Ser199Phe (596C/T) polymorphism, PCR products of segment $2 \mathrm{c}$ were digested overnight with $\mathrm{Mbo}$ II at $37^{\circ} \mathrm{C}$, leaving a 401-bp fragment for the $T$ allele, and 234- and 167-bp fragments for the $C$ allele. For the $-983 C / T$ polymorphism, genotyping was performed by PCR products using primers as follows: forward, 5'-TGGGATCTATGGGGGTGGGGTTCTAAGTGC-3', reverse, 5'-AGAGAGATCCTGCCTATGTCCCTTCCATGC-3'. Each primer contained one nucleotide mismatch (underlined), which made it possible to use the restriction enzyme Hha I for the detection of the $-983 C / T$ polymorphism. PCR was carried out in a $20-\mu 1$ reaction mixture described above, added with $5 \%$ DMSO. For this primer set, initial denaturation at $94^{\circ} \mathrm{C}$ for $5 \mathrm{~min}, 35$ cycles of $30 \mathrm{sec}$ at $94^{\circ} \mathrm{C}$, $30 \mathrm{sec}$ at $65^{\circ} \mathrm{C}$ were run, followed by an additional $7 \mathrm{~min}$ at $72^{\circ} \mathrm{C}$ after the last cycle. PCR products were digested overnight with Hha $\mathrm{I}$ at $37^{\circ} \mathrm{C}$, leaving a 96- and 29-bp fragments for the $C$ allele, and 125-bp fragment for the $T$ allele. Genotypes were determined by electrophoresis on $3 \%$ agarose gel and ethidium bromide staining. The -43 ins $C A$ and $-1822 G / A$ polymorphisms were screened by direct sequencing, because these polymorphisms did not alter any restriction enzyme recognition site.

\section{Results}

We identified two polymorphisms of the $n g n 3$ gene, a substitution of $\mathrm{C}$ to $\mathrm{T}$ at position 596, which was a missense variant which converts codon 199 Ser to Phe, and an insertion of CA at position -43 , which lies in intron 1, at a position 42-bp upstream of the junction between intron 1 and exon 2 . We also identified two polymorphisms in the upstream region, a substitution of $\mathrm{C}$ to $\mathrm{T}$ at position -983 and a substitution of $\mathrm{G}$ to $\mathrm{A}$ at position -1822 . The allelic frequencies of these polymorphisms are shown in Table 2. The frequencies of both the alleles were in HardyWeinberg equilibrium. There is linkage disequilibri- 
Table 2. Polymorphisms of the ngn 3 gene



um among these four polymorphisms with pairwise linkage disequilibrium coefficient D' values $[8,9]$ in Type II diabetic patients of Ser199Phe and -43insCA, 0.76; Ser199Phe and -983C/T, 0.72; Ser199Phe and $-1822 G / A,-0.64$; -43 ins $C A$ and $-983 C / T, 0.97$; -43 in$s C A$ and $-1822 G / A,-0.51$; and $-983 C / T$ and $-1822 G /$ $A,-0.46$. Similar D' values were observed in control subjects. Analysis of the distribution of the two alleles using a simple chi-squared $\left(\chi^{2}\right)$ test revealed no association between these genotypes and Type II diabetes. Among the diabetic patients, we did not find any significant difference in terms of treatment between those with and without the polymorphisms. The allelic frequencies of the Ser199Phe polymorphism in patients who were treated with insulin, oral hypoglycaemic agents and diet alone were $0.736,0.727$ and 0.691 for $P h e$, respectively, those of the $-43 i n s C A$ polymorphism in the same groups of patients were 0.934 , 0.912 and 0.894 for the insertion allele, respectively, those of the $-983 C / T$ polymorphism in the same groups of patients were $0.943,0.912$ and 0.904 for the $C$ allele, respectively, and those of the $-1822 G / A$ polymorphism in the same groups of patients were 0.538 , 0.526 and 0.532 for the $G$ allele, respectively.

We also identified differences between the sequences reported in GenBank and those in our subjects at three sites in the coding region. Although the nucleotides at positions 128, 292 and 493 reported in GenBank were $C, G$ and $C$, respectively, our data demonstrated that these corresponding nucleotides were $G, A$ and $T$, respectively, in all of the 81 patients with Type II diabetes and 20 control subjects examined by us.

\section{Discussion}

We screened for variability of the ngn 3 gene in 197 patients with Type II diabetes and 216 control subjects. This showed one missense variant at codon 199 (Ser $\rightarrow$ Phe) and one insertion-deletion variant in intron 1. We also identified two polymorphisms in the upstream region. The allelic frequencies of these polymorphisms were not statistically significantly different between Type II diabetic patients and control subjects. The variants were also not associated with treatments among diabetic patients. We concluded that the Ser199Phe, -43 ins $C A,-983 C / A$ and $-1822 G /$ $A$ polymorphisms of $n g n 3$ are not significantly related to either the development or the severity of Type II diabetes.

The differences between the sequences reported in GenBank and those in our subjects were associated with changes of amino acids. One of these changes was present at the codon 98, which was in the junctional region between the basic region and the helix 1 region of the bHLH domain [5]. We cannot exclude the possibility that the differences between our data and those submitted data in GenBank reflect the differences between ethnic groups.

We identified four polymorphisms of the ngn3 gene and determined that these polymorphisms were not significantly associated with Type II diabetes in the Japanese subjects. We cannot exclude the possibility that these polymorphisms contribute to the development of Type II diabetes in combination with other factors. Rare sequence variants other than those presented by us here could play a part in the 
pathogenesis of MODY (maturity-onset diabetes of the young)-like diabetes, idiopathic Type I diabetes or neurological disorders, since ngn3 is involved in the development of both of the endocrine cells of the pancreas and the nervous system [5]. These polymorphisms could be useful for genetic studies by the haplotype analysis approach. Further studies are needed to evaluate the possible biological impact of variants of the ngn 3 gene on the pathogenesis of diabetes.

Acknowledgements. This study was supported by a Grant-inAid for creative basic research (10NP0201) from the Ministry of Education, Science, Sports and Culture, Japan (to T. Kadowaki).

\section{References}

1. Fajans SS, Bell GI (2000) Maturity-Onset Diabetes of the Young. In: LeRoith D, Taylor SI, Olefsky JM (eds) Diabetes Mellitus. 2nd edn. Lippincott Williams \& Wilkins, Philadelphia, pp 691-705

2. Macfarlane WM, Frayling TM, Ellard S et al. (1999) Missense mutations in the insulin promoter factor- 1 gene predispose to type 2 diabetes. J Clin Invest 104: R33-R39
3. Hani EH, Stoffers DA, Chevre JC et al. (1999) Defective mutations in the insulin promoter factor-1 (IPF-1) gene in late-onset Type II diabetes mellitus. J Clin Invest 104: R41R48

4. Malecki MT, Jhala US, Antonellis A et al. (1999) Mutations in NEUROD1 are associated with the development of Type II diabetes mellitus. Nat Genet 23: 323-328

5. Sommer L, Ma Q, Anderson DJ (1996) Neurogenins, a novel family of atonal-related bHLH transcription factors, are putative mammalian neuronal determination genes that reveal progenitor cell heterogeneity in the developing CNS and PNS. Mol Cell Neurosci 8: 221-241

6. Gradwohl G, Dierich A, LeMeur M, Guillemot F (2000) Neurogenin 3 is required for the development of the four endocrine cell lineages of the pancreas. Proc Natl Acad Sci USA 97: 1607-1611

7. Alberti KGMM, Zimmet PZ for the WHO Consultation (1998) Definition, Diagnosis and Classification of Diabetes Mellitus and its Complications Part 1: Diagnosis and Classification of Diabetes Mellitus Provisional Report of a WHO Consultation. Diabet Med 15: 539-553

8. Jorde LB, Watkins WS, Carlson M et al. (1994) Linkage disequilibrium predicts physical distance in the adenomatous polyposis coli region. Am J Hum Genet 54: 884-898

9. Long JC, Wiliams RC, Urbanek M (1995) An E-M algorithm and testing strategy for multiple-locus haplotypes. Am J Hum Genet 56: 799-810 